\title{
Funky Grooves: Declarative Programming of Full-Fledged Musical Applications
}

\author{
Henrik Nilsson ${ }^{1}$ and Guerric Chupin ${ }^{2}$ \\ 1 School of Computer Science, University of Nottingham, Nottingham, UK, \\ nhn@cs.nott.ac.uk, \\ 2 ENSTA ParisTech, Palaiseau, France, \\ guerric.chupin@ensta-paristech.fr
}

\begin{abstract}
There are many systems and languages for music that essentially are declarative, often following the synchronous dataflow paradigm. As these tools, however, are mainly aimed at artists, their application focus tends to be narrow and their usefulness as general purpose tools for developing musical applications limited, at least if one desires to stay declarative. This paper demonstrates that Functional Reactive Programming (FRP) in combination with Reactive Values and Relations (RVR) is one way of addressing this gap. The former, in the synchronous dataflow tradition, aligns with the temporal and declarative nature of music, while the latter allows declarative interfacing with external components as needed for full-fledged musical applications. The paper is a case study around the development of an interactive cellular automaton for composing groove-based music.
\end{abstract}

Keywords: functional reactive programming, reactive values and relations, synchronous dataflow, hybrid systems, music

\section{Introduction}

Time, simultaneity, and synchronisation are all inherent aspects of music. Further, there is much that is declarative about music, such as musical notation and many underpinning aspects of music theory. This suggests that a timeaware, declarative paradigm like synchronous dataflow [5] might be a good fit for musical applications. Indeed, there are numerous successful examples of languages and systems targeting music that broadly fall into that category, such as CSound ${ }^{3}, \operatorname{Max} / \mathrm{MSP}^{4}$, and Pure Data ${ }^{5}$ just to mention three.

However, systems like these primarily target artists and are not in themselves general purpose languages. It may be possible to extend them to support novel applications, but this usually involves non-declarative programming and working around limitations such as lack of support for complex data structures [7, p.170] or difficulties to express dynamically changing behaviour [7, p.156][1].

\footnotetext{
${ }^{3}$ http://www. csounds.com/

${ }^{4}$ https://cycling74.com/products/max/

${ }^{5}$ https://puredata.info/
} 
With this application paper, we aim to demonstrate that Functional Reactive Programming (FRP) $[8,13]$ in combination with Reactive Values and Relations (RVR) [15] is a viable and compelling approach to developing full-fledged musical applications in a declarative style, and, by extension, other kinds of interactive applications where time and simultaneity are central. To cite Berry [2]:

From the points of view of modeling and programming, there is actually not much difference between programming an airplane or an electronic orchestra.

A more detailed account of this work is available as a technical report [12].

FRP combines the full power of polymorphic functional programming with synchronous dataflow, thus catering for the aforementioned temporal aspects while not being restricted by being tied to any specific application domain. Its suitability for musical applications has been demonstrated a number of times. For example, it constitutes an integral part of the computer music system Euterpea ${ }^{6}$, which supports a broad range of musical applications [10], and it has been used for implementing modular synthesizers [9].

Generally, though, the core logic is only one aspect of a modern, compelling software application. In particular, musical applications usually require sophisticated, tailored GUIs and musical I/O, such as audio or MIDI. In practice, such requirements necessitate interfacing with large, complex, and often platformspecific imperative frameworks. In contrast to earlier work [9], we do consider external interfacing here: RVR was developed specifically to meet that need in a declarative manner.

The paper constitutes a case study of the development of a medium-sized musical application inspired by the reacTogon [4], an interactive (hardware) cellular automaton for groove-based music. The FRP system used is Yampa [13]. To challenge our frameworks, we have adapted and extended the basic idea of the reacTogon considerably to create a useful and flexible application that fits into a contemporary studio setting. Through an overview of the developed application and highlights of techniques and code fragments, we hope to convince the reader that our approach works in practice for real applications and has many merits. The source code for the application is publicly available on GitLab ${ }^{7}$.

\section{Background}

\subsection{Time in Music}

Change over time is an inherent aspect of music. Further, at least when considered at some level of abstraction, such as a musical score or from the perspective of music theory, music exhibits both discrete-time, and continuous-time aspects [6, p.127]. In music theory, this is referred to as striated and smooth time, a distinction usually attributed to the composer Pierre Boulez [3]. For example,

\footnotetext{
${ }^{6}$ http://www . euterpea.com/

7 https://gitlab.com/chupin/arpeggigon
} 
the notes in a musical score begin at discrete points in time. On the other hand, crescendo is the gradual increase of the loudness, ritardando is the gradual decrease of the tempo, and portamento is the gradual change of the pitch from one note to another. Contemporary electronic musical genres provide many other examples of gradual change as an integral part of the music, such as smooth filter sweeps or rhythmic changes of the volume.

Of course, there are many more aspects of time in music than discrete vs. continuous [6, pp.123-130]. However, for musical applications, support for developing mixed discrete- and continuous-time systems, often referred to as hybrid systems, is a good baseline.

\subsection{Functional Reactive Programming and Yampa}

Functional Reactive Programming (FRP) [8] is a declarative approach to implementing reactive applications centred around programming with time-varying values in the synchronous dataflow tradition [5]. In this paper, we are using the arrows-based [11] FRP system Yampa [13]. It is realised as an embedding in Haskell and it supports hybrid systems whose structure may change over time. Thus, as discussed in Sect. 2.1, it is a good fit for musical applications. Further, the arrows-based programming model is close to the visual "boxes and arrows" approach. This also goes well with musical applications, as evidenced by systems like Max/MSP and similar. We outline some of the basic aspects of Yampa in the following for the benefit of readers not familiar with it. A more in-depth account can be found in e.g. the accompanying technical report [12].

Yampa is based on two central concepts: signals and signal functions. A signal is a function from time to values of some type:

$$
\text { Signal } \alpha \approx \text { Time } \rightarrow \alpha
$$

Time is (notionally) continuous, represented as a non-negative real number. (We will return to discrete time shortly.) The type parameter $\alpha$ specifies the type of values carried by the signal. A signal function is a function from Signal to Signal:

$$
\text { SF } \alpha \beta \approx \text { Signal } \alpha \rightarrow \text { Signal } \beta
$$

When a value of type $S F \alpha \beta$ is applied to an input signal of type Signal $\alpha$, it produces an output signal of type Signal $\beta$. Signal functions are first class entities in Yampa. Signals, however, are not: they only exist indirectly through the notion of signal function.

Programming in Yampa consists of defining signal functions compositionally using Yampa's library of primitive signal functions and a set of combinators. Some central arrow combinators are arr that lifts an ordinary function to a stateless signal function, serial composition $\ggg$, parallel composition $\&$, and the fixed point combinator loop. Figure 1 illustrates these combinators pictorially. In practice, Paterson's arrow notation [14] is often used to facilitate writing arrow code. It is a variation of Haskell's do-notation and essentially allows diagrams to be described textually by naming the arrows. 


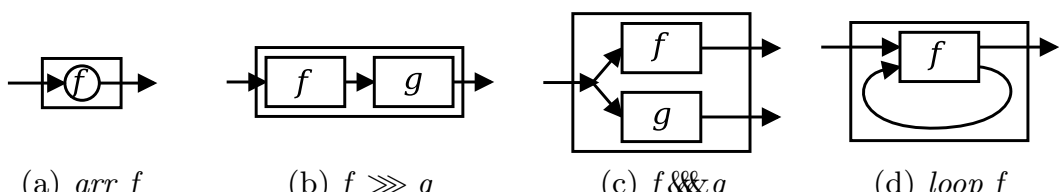
(a) $\operatorname{arr} f$
(b) $f \ggg g$
(c) $f \& \& g$
(d) $\operatorname{loop} f$

Fig. 1. Basic signal function combinators.

The Event type models discrete-time signals:

data Event $a=$ NoEvent $\mid$ Event $a$

A signal function whose output signal is of type Event $T$ for some type $T$ is called an event source. The value carried by an event occurrence may be used to convey information about the occurrence.

A family of switching primitives enable the system structure to change in response to events. The simplest such primitive is switch:

$$
\text { switch }:: \text { SF } a(b, \text { Event } c) \rightarrow(c \rightarrow S F a b) \rightarrow S F a b
$$

Once the switching event occurs, switch applies its second argument to the value carried by the event and switches into the resulting signal function. Yampa also includes parallel switching constructs that maintain dynamic collections of signal functions connected in parallel [13].

\subsection{Reactive Values and Relations}

A Reactive Value (RV) [15] is a typed mutable value with access rights and change notification. RVs provide a light-weight and uniform interface to GUI widgets and other external components such as files and network devices. Each entity is represented as a collection of RVs, each of which encloses an individual property. RVs can be transformed and combined using a range of combinators, including lifting of pure functions and lenses.

Reactive Relations (RR) specify how RVs are related separately from their definitions. An RR may be uni- or bi-directional. Once RVs have been related, changes will be propagated automatically among them to ensure that the stated relation is respected.

\section{The Arpeggigon}

Our application is called Arpeggigon, from arpeggio and hexagon. It was inspired by Mark Burton's hardware reacTogon: a "chain reactive performance arpeggiator" [4]. However, we have expanded considerably upon the basic idea to create a software application we believe is both genuinely useful in a contemporary studio setting and a credible test case for our approach. 


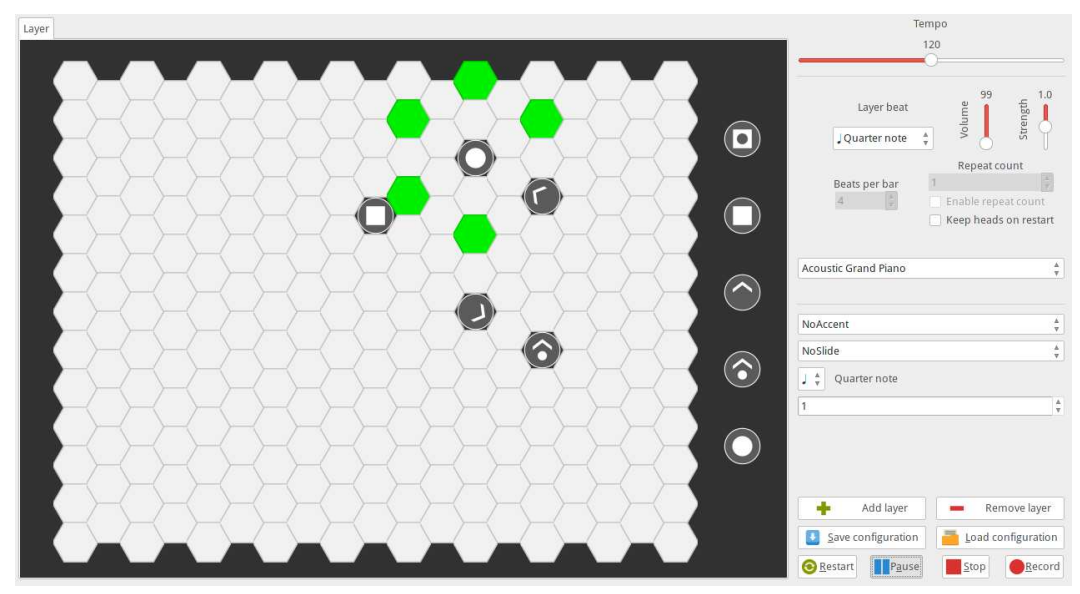

Fig. 2. The Arpeggigon

\subsection{The reacTogon}

Central to the design of the reacTogon is the Harmonic Table ${ }^{8}$ : a way to arrange musical notes on a hexagonal grid. The various directions correspond to different musically meaningful intervals. For example, each step along the vertical axis corresponds to a perfect fifth. The reacTogon uses this layout to implement a cellular automaton. See Fig. 2 for our adaptation of the idea. Tokens of a few different kinds are placed on the grid, at most one token per cell. These tokens govern how play heads move around the grid, as well as the initial position and direction of the play heads. When a play head hits a token, the kind of token determines what happens next. First, for most tokens, a note corresponding to the position of the token is played. Second, either the direction of the play head is changed, it is split into new play heads, or it is absorbed. Thus, arpeggiated chords or other sequences of notes are described. These can further be transposed in response to playing a keyboard, allowing the reacTogon to be performed.

\subsection{Features and Architecture}

Our Arpeggigon is a software realization of the reacTogon concept. The main features our Arpeggigon provides over the reacTogon are:

- Multiple layers: one or more cellular automata run in parallel. Layers can be added, removed, and edited dynamically through a tabbed GUI.

- Extended attributes for tokens, such as note length, accent, and slide.

- Per-cell repeat count for local modification of the topology of the grid.

- MIDI integration.

- Saving and loading of configurations.

\footnotetext{
${ }^{8}$ https://en.wikipedia.org/wiki/Harmonic_table_note_layout
} 


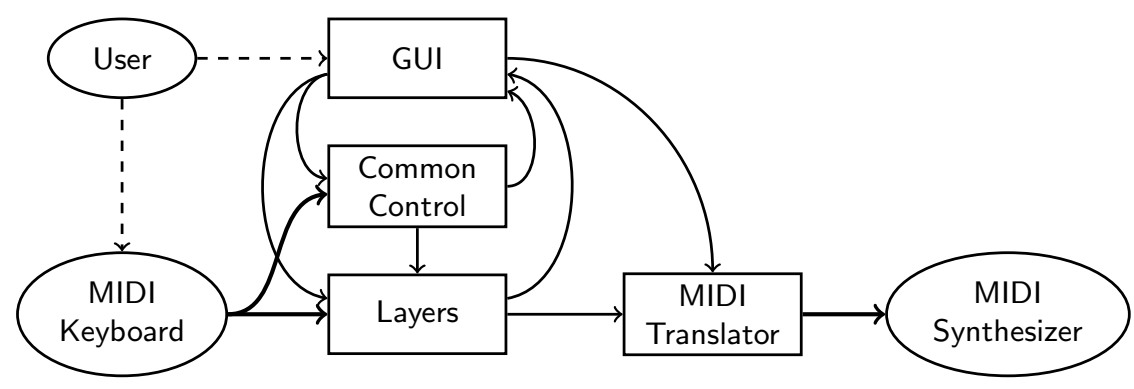

Fig. 3. The Arpeggigon architecture

Figure 2 shows a screenshot. Dynamic addition and removal of layers means that both the core logic of the application and the GUI must support structural changes while the application is running. Note the different kinds of tokens to the right of the grid. They can be dragged and dropped onto the grid to configure a layer, even while the Arpeggigon is running. The play heads are coloured green.

Figure 3 illustrates the architecture of the Arpeggigon. The rectangles represent the main system components. The thin arrows represent internal communication, the thick ones MIDI I/O, and the dashed ones user interaction.

GUI is the graphical user interface. It includes a model of the state of global parameters, such as the overall system tempo, and the current configuration of each layer. Common Control is responsible for system-wide aspects, such as generating a global clock (reflecting the system tempo) that keeps the layers synchronised. Layers is the instances of the actual automata, each generating notes. MIDI Translator translates high-level internal note events and control signals into low-level MIDI messages, merging and serialising the output from all layers.

GUI communicates the current system configuration to Common Control and Layers. Note that this data is time-varying as the user can change the configuration any time. Layers needs to communicate the positions of the play heads back to GUI for animation purposes. This is thus also a time-varying signal.

\section{Implementation}

\subsection{Layers}

At its core, each layer of the Arpeggigon is a cellular automaton that advances one step per layer beat. Its semantics is embodied by a transition function:

$$
\begin{gathered}
\text { advanceHeads }:: \text { Board } \rightarrow \text { BeatNo } \rightarrow \text { RelPitch } \rightarrow \text { Strength } \rightarrow[\text { PlayHead }] \\
\rightarrow([\text { PlayHead }],[\text { Note }])
\end{gathered}
$$

In essence, given the current configuration of tokens on the hexagonal grid, henceforth the board, it maps the state of the play heads (position, direction, and a repeat counter) to an updated play head state and a list of notes to be played at this beat. The number of play heads may change as a play head may 
be split or absorbed. The remaining parameters give the current transposition of the layer, the strength with which notes should be played, and the beat number within a bar allowing specific notes in a bar to be accented (played stronger).

Using the scanl-like Yampa function accumBy, advanceHeads is readily lifted into an event-processing signal function:

$$
\begin{aligned}
\text { automaton }::[\text { PlayHead }] \rightarrow S F & (\text { Board, DynamicLayerCtrl, Event BeatNo }) \\
& (\text { Event }[\text { Note }],[\text { PlayHead }])
\end{aligned}
$$

The static parameter is the initial state of the play heads. The first of the three input signals carries the current configuration of the board, originating from GUI (Fig. 3). The second carries a record of dynamic control parameters for the layer, including transposition, play strength, and the length of a layer beat, originating from GUI and MIDI Keyboard. These two are continuous-time signals, reflecting the fact that the configuration of the board can change and a key be struck on the MIDI keyboard at any time, not just at a beat. The third is the discrete-time layer beat clock, from Common Control, carrying the beat number within a bar. The output signals are the notes to be played, to be sent to MIDI Translator, and the state of the play heads for animation purposes, to be sent back to GUI. Note the close correspondence to the architecture in Fig. 3.

\subsection{Synchronisation}

As an example of turning Yampa's continuous-time capabilities to musical applications, consider automating gradual tempo changes. Imagine two sliders to set a fast and a slow tempo, a button to select between them, and a further slider to set the rate at which the tempo should change. The following signal function derives a smoothly changing tempo from these controls, regulated to within $0.1 \mathrm{bpm}$ of the desired tempo. Note the feedback (enabled by rec):

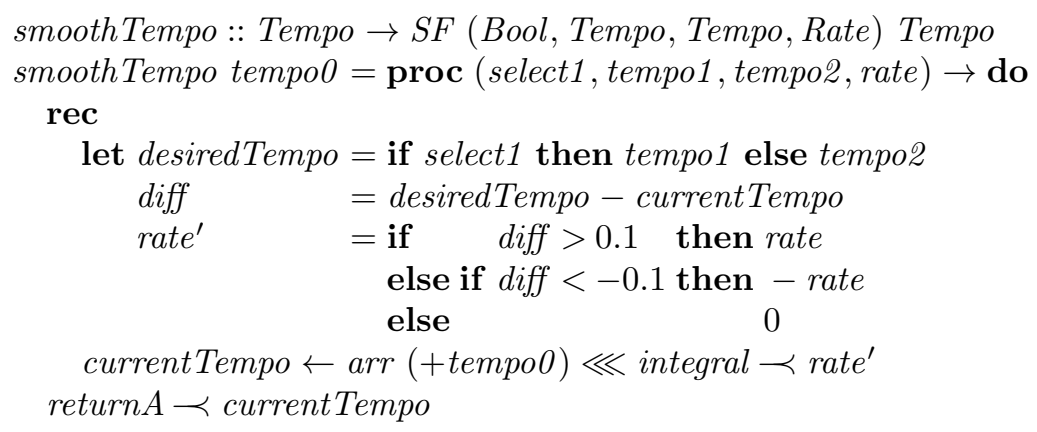

\subsection{GUI and Interaction}

The GUI of the Arpeggigon is written using the cross-platform widget toolkit GTK + . The Arpeggigon does not generate any audio by itself; it needs to be connected to an external, MIDI-capable hardware or software synthesizer. MIDI $\mathrm{I} / \mathrm{O}$ is handled by the JACK Audio Connection Kit. 
All code for interfacing with the external world is structured using reactive values and relations (RVR). Much of this code is of course monadic (in the IO monad). However, as it is mostly concerned with creating and interconnecting interface entities, the code has a fairly declarative reading as a sequence of entity definitions and specifications of how they are related.

As a case in point, consider the following code for the system tempo slider:

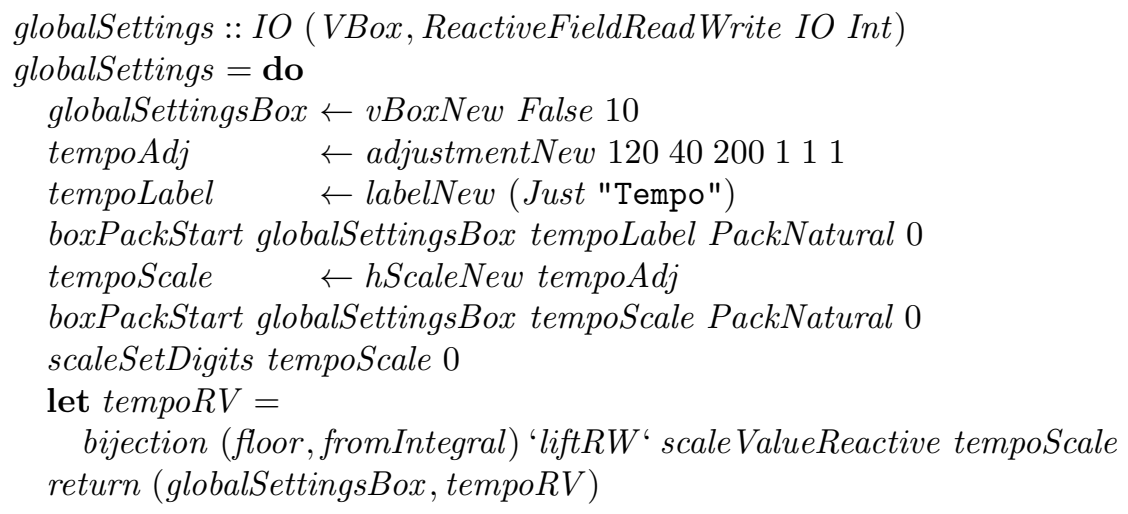

In essence, this code defines a box, a label, and a slider, and visually relates them by placing the last two inside the box. This is all standard GTK+. A $\mathrm{read} /$ write, integer-valued reactive value $(\mathrm{RV})$ is finally defined and related to the real-valued value of the slider: scaleValueReactive associates a slider with an RV, while liftRW derives a new RV from an existing one by specifying two conversion functions, one for reading and one for writing.

Finally, the RVR part and the Yampa part of the Arpeggigon are connected by the following function:

$$
\begin{aligned}
& \text { yampaReactiveDual :: } \\
& \qquad a \rightarrow \text { SF } a b \rightarrow \text { IO (ReactiveFieldWrite IO a, ReactiveFieldRead IO } b)
\end{aligned}
$$

This creates two reactive values: one for the input and one for the output of the signal function. After writing a value to the input, the corresponding output at that point in time can be read.

\section{Conclusions}

This paper demonstrated how Functional Reactive Programming in combination with Reactive Values and Relations can be used to develop a realistic, non-trivial musical application. On the whole, we found that these two frameworks together were very well suited for this task. The performance was good, including critical aspects like jitter, without much effort so far having been spent on optimisation. Heap usage and overall memory footprint was modest. See the accompanying technical report for details [12]. Further, as most of the techniques we demonstrated are not limited to a musical context, we suggest that this is a good approach for programming time-aware, interactive applications in general. 
Acknowledgments. The authors would like to thank Ivan Perez and Henning Thielemann for support and advice with the reactive libraries and the Haskell JACK bindings respectively, Michel Mauny for co-supervising the second author's summer internship with the Functional Programming Laboratory in Nottingham, and François Pessaux and anonymous reviewers for helpful feedback.

\section{References}

1. Guillaume Baudart, Louis Mandel, and Marc Pouzet. Programming mixed music in ReactiveML. In 1st Workshop on Functional Art, Music, Modeling and Design (FARM), pages 11-22, Boston, USA, September 2013. ACM.

2. Gerard Berry. Formally unifying modeling and design for embedded systems A personal view. In 7th International Symposium on Leveraging Applications of Formal Methods, Verification and Validation (ISoLA), Part II, number 9953 in Lecture Notes in Computer Science, pages 134-149. Springer, 2016.

3. Pierre Boulez. Penser la musique aujourd'hui. Gallimard, 1964.

4. Mark Burton. The reacTogon: a chain reactive performance arpeggiator. https://www . youtube. com/watch?v=AklKy2NDpqs, 2007.

5. Paul Caspi, Daniel Pilaud, Nicolas Halbwachs, and John A. Plaice. LUSTRE: A declarative language for programming synchronous systems. In 14th Symposium on Principles of Programming Languages (POPL), New York, NY, 1987. ACM.

6. Arshia Cont. Antescofo: Anticipatory synchronization and control of interactive parameters in computer music. In International Computer Music Conference (ICMC), pages 33-40, Belfast, Ireland, August 2008.

7. Arshia Cont. Modeling Musical Anticipation: From the Time of Music to Music of Time. PhD thesis, University of California San Diego (UCSD) and University of Pierre et Marie Curie (Paris VI), 2008.

8. Conal Elliott and Paul Hudak. Functional reactive animation. In 2nd International Conference on Functional Programming (ICFP), pages 163-173, June 1997.

9. George Giorgidze and Henrik Nilsson. Switched-on Yampa: Declarative programming of modular synthesizers. In Practical Aspects of Declarative Languages (PADL) 2008, volume 4902 of Lecture Notes in Computer Science, pages 282-298, San Francisco, CA, USA, January 2008. Springer-Verlag.

10. Paul Hudak, Donya Quick, Mark Santolucito, and Daniel Winograd-Cort. Realtime interactive music in Haskell. In 3rd International Workshop on Functional Art, Music, Modelling and Design (FARM), pages 15-16, Vancouver, BC, Canada, September 2015. ACM.

11. John Hughes. Generalising monads to arrows. Science of Computer Programming, 37:67-111, May 2000.

12. Henrik Nilsson and Guerric Chupin. The Arpeggigon: Declarative programming of a full-fledged musical application. Technical Report, http://eprints.nottingham.ac.uk/38657, November 2016.

13. Henrik Nilsson, Antony Courtney, and John Peterson. Functional reactive programming, continued. In Haskell Workshop, pages 51-64, Pittsburgh, PA, USA, October 2002. ACM.

14. Ross Paterson. A new notation for arrows. In International Conference on Functional Programming (ICFP), pages 229-240, Firenze, Italy, September 2001.

15. Ivan Perez and Henrik Nilsson. Bridging the GUI gap with reactive values and relations. In 8th ACM SIGPLAN Symposium on Haskell, pages 47-58, Vancouver, Canada, 2015. ACM. 